\title{
A New Dynamic Structure Neural Network for Control of Nonlinear Systems
}

\author{
Mahdi Jalili-Kharaajoo \\ Young Researchers Club, Azad University, Tehran, Iran \\ mahdijalili@ece.ut.ac.ir
}

\begin{abstract}
In this paper, Dynamic structure neural network controller based on feedback linearization is proposed. The proposed method can adapt the neural network structure dynamically while it can guarantee the stability and tracking precision of system.
\end{abstract}

\section{Introduction}

Neural network provides a nice form for describing the nonlinear characteristic of systems in certain degree and it has the vast vistas in the control of nonlinear systems $[1,2,3]$. There is a strong appeal to the research field of nonlinear control theory and neural network control if combining neural network with nonlinear adaptive control $[4,5]$. But there is the short of systematic study on the stability and robust property for the nonlinear neural network control system so far. In this note, we will propose an efficient approach to combine the dynamic structure neural network algorithm with adaptive feedback linearization. The algorithm of dynamic structure neural network controller is given and the stability of system is analyzed. It is satisfactory with the results of system simulation.

\section{Dynamic Structure Neural Network Controller}

Supposing the nonlinear system (SISO) is described

$$
\begin{aligned}
& \dot{X}=f(X)+g(X) u \\
& y_{p}=h(X)
\end{aligned}
$$

Where $X \in R^{n}, y_{p}$ and $u$ are the state, output and control input variables respectively. If the order and relative degree of system (1) are $n$ and $r$, then we let

$$
u=\frac{1}{g_{1}(X)}\left(-f_{1}(X)+v\right)
$$

Where $v$ is pseudo-input, $f_{1}=L_{f}^{r} h(X), g_{1}=L_{g} L_{f}^{r-1} h(X)$. ( $L \quad$ is Lee derivative operator)[6]. The original system can be changed into linear system and

$$
y^{r}=v
$$

Generally, the objective of control system for (1) is to find the control law $u$ so that the outputs of system follow the reference trajectory $y_{m}$. The relative degree of reference model $n$ is greater than $r$. For minimum-phase system, we let 


$$
v=y_{m}^{r}-\alpha_{r} e^{r-1}-\ldots-\alpha_{1} e
$$

where $e=y_{p}-y_{m}$ and $\alpha_{r}, \alpha_{r-1}, \ldots, \alpha_{1}$ satisfy $\Gamma(s)=\left(s^{r}+\alpha_{r} s^{r-1}+\ldots+\alpha_{r}\right) \quad$ is Hurwitz polynomial. In order to guarantee the stability and tracking precision of system, we take the control law

$$
u=u_{a l}+u_{s l}, u_{a l}=\frac{1}{\hat{g}(X)}(-\hat{f}(x)+v)
$$

and $u_{s l}$ is sliding controller.

$f(X), g(X)$ are the approaches of $f_{1}(X), g_{1}(X)$ respectively. Using the method of [1], we let

$$
\hat{f}(X)=m(X) \hat{W}_{f}^{T} \Phi_{f}(X)+f_{o}(X), \hat{g}(X)=m(X) \hat{W}_{g}^{T} \Phi_{g}(X)+g_{o}(X)
$$

where $\Phi_{f}(X)$ and $\Phi_{g}(X$.$) are the output of hidden layers of neural network and \hat{W}_{f}$, $\hat{W}_{g}$ are the parameters of corresponding output layers.

Using the method of feedback linearization, the state locus of system is in the subspace $A_{d}$ of the state space. It can be decided by reference model. Neural network is only employed to approach the characteristic of system in subspace $A_{d}$. To ensure safety we expand the approximation area to $A$ where $A_{d} \subset A$. The $m(x)$ expresses the switch function to control neural network so that neural network only approaches the characteristic of system in subspace $A$. It is defined as

$$
m(X)=\left\{\begin{array}{lc}
1 & X \in A_{d} \\
0-1 & X \in\left(A-A_{d}\right) \\
0 & X \notin A_{d}
\end{array}\right.
$$

The $f_{o}, g_{o}$ are the known characteristic of $f_{1}, g_{1}$ receptively.

According to the approaching theory of neural network, we have:

Supposition (1) for any $\varepsilon_{f}, \varepsilon_{g} \in R^{+}$and $f_{o}, g_{o}$ there exist $W_{f}^{*}, W_{g}^{*}$ and $m(X)$ so that for any $x \in A$ in (6) satisfy

where

$$
|\Delta f|=\left|f^{*}(X)-f_{1}(X)\right| \leq \varepsilon_{f},|\Delta g|=\left|g^{*}(X)-g_{1}(X)\right| \leq \varepsilon_{g}
$$

$$
f^{*}=\hat{f}\left(W_{f}^{*}, X\right), \Delta f=f^{*}(X)-f_{1}(X), g^{*}=\hat{g}\left(W_{g}^{*}, X\right), \Delta g=g^{*}(X)-g_{1}(X)
$$

The neurons in dynamic structure neural network can be regarded as to be chosen from the set of candidate neural neurons. The set of all candidate neurons is $K_{p o t}$ which is determined according to the properties of hidden layer neurons and the property of system. The neurons in $K_{\text {pot }}$ can be divided into three sets, 1) inactive neurons $K_{p} ; 2$ ) active neurons to be removed from neural network $K_{d}$; 3) active neurons to be kept in the neural networks $K a$. Then, we have

$$
f(X)=m W_{f}^{*} \Phi_{f}-\Delta f+f_{o}=m\left[\begin{array}{c}
W_{f a}^{*} \\
W_{f d}^{*} \\
W_{f p}^{*}
\end{array}\right]^{T}\left[\begin{array}{l}
\Phi_{f a} \\
\Phi_{f d} \\
\Phi_{f p}
\end{array}\right]-\Delta f+f_{o}, g(X)=m W_{g}^{*} \Phi_{g}-\Delta g+g_{o}=m\left[\begin{array}{c}
W_{g a}^{*} \\
W_{g d}^{*} \\
W_{g p}^{*}
\end{array}\right]^{T}\left[\begin{array}{l}
\Phi_{g a} \\
\Phi_{g d} \\
\Phi_{g p}
\end{array}\right]-\Delta g+g_{o}
$$

Let

$$
e_{1}=\beta_{r} e^{r-1}+\beta_{r-1} e^{r-2}+\ldots+\beta_{1}
$$


where $\beta_{r}, \ldots, \beta_{1}$ satisfy $\varphi(s)=\beta_{r} s^{r-1}+\beta_{r-1} s^{r-2}+\ldots+\beta_{1}$ is Hurwitz polynomial and $\varphi(s) / \Gamma(s)$ is strict positive real function.

We can choose $\varphi(s), \Gamma(s)$ such that

$$
\varphi(s) / \Gamma(s)=\frac{1}{s+k_{d}}, k_{d}>0
$$

In order to avoid the parameters drift, increase the robustness of the system, and add dead-zone to $e_{1}$, Let

$$
e_{1 \Delta}=e_{1}-\phi \operatorname{sat}\left(\frac{e_{1}}{\phi}\right)
$$

and take the sliding mode control as

$$
u_{s l}=-k_{s l} \operatorname{sat}\left(\frac{e_{1}}{\phi}\right)
$$

The adaptive rate of neural network will be taken as

$$
\begin{gathered}
\frac{d \hat{W}_{f a}}{d t}=\eta_{f} m e_{1 \Delta} \Phi_{f a}, \frac{d \hat{W}_{f d}}{d t}=\eta_{f} m\left|e_{1 \Delta}\right|\left[-\operatorname{sgn}\left(\hat{W}_{f d}\right)\right], \frac{d \hat{W}_{f p}}{d t}=0, \frac{d \hat{W}_{g a}}{d t}=\eta_{g} m e_{1 \Delta} \Phi_{g a} \\
\frac{d \hat{W}_{g d}}{d t}=\eta_{g} m\left|e_{1 \Delta}\right|\left[-\operatorname{sgn}\left(\hat{W}_{g d}\right)\right], \frac{d \hat{W}_{g p}}{d t}=0
\end{gathered}
$$

\section{Simulation Results}

Suppose that a nonlinear system is described as

$$
\begin{aligned}
& \dot{x}_{1}=f(X)+g(X) u \\
& \dot{x}_{2}=x_{1}-x_{2} \\
& y_{p}=x_{1}
\end{aligned},\left\{\begin{array}{l}
X=\left[\begin{array}{l}
x_{1} \\
x_{2}
\end{array}\right] \\
f(X)=-4 \frac{\sin \left(4 \pi x_{1}\right)}{\left(4 \pi x_{1}\right)} \frac{\left(\sin \left(4 \pi x_{2}\right)\right)^{2}}{\left(4 \pi x_{2}\right)^{2}}, g(X)=2+\cos \left(7 x_{1} x_{2}\right)
\end{array}\right.
$$

and system order $n=2$, relative degree $r=1$.

The reference model is $y_{d}=1 /(1+s / 10)^{3}$. and its input is the square wave with 0.4 Hz frequency and 0.9 amplitude. Let $\beta_{1}=1, \alpha_{r}=k_{d}=5, \phi=0.005$.

From the reference model, we can know that the state locus of system is in the region of $[-1,1] *[-1,1]$. So, we can take $A_{d}=[-1,1] \times[-1,1], A=[-1.5,1.5] \times[-1.5,1.5]$.

In system simulation, the neural network controller based on dynamic structure wavelet network is employed. The 2-d mother wavelet $\psi(X)=\left(2-\|X\|^{2}\right) \exp \left(-\|X\|^{2} / 2\right)$. And the corresponding 2-D wavelet frame is $\psi_{p, K}(X)=a_{o}{ }^{-p n / 2} \psi\left(a_{o}{ }^{-p} X-\operatorname{diag}(K) b_{o}\right)$. Where $p \in Z, K \in Z_{0}^{2}$ are the scale and translation coefficients. And $a_{o}=2, b_{o}=1$, $p \in[-1,4]$. The range of $K$ is chosen so that $a_{o}{ }^{p} b_{o} K \in A$.

The adaptive rate of neural network $\eta_{f}=\eta_{g}=25$. The structure adaptive law coefficients are $u_{f}=1, u_{g}=5, \theta_{f}=3, \theta_{g}=15, f_{o}=0, g_{o}=1.9, g_{l}=0.895$. The sliding mode controller is $k_{s l}=0.5$ and the initial states of system are $X(0)=[1,1]^{T}$. 
The simulated results are given in Figs. 1 and 2. It shows that the neural network structure is dynamic and tending towards stable and the tracking error of system is reaching the required zone after 10 seconds. The neural network sizes for approximating $f(X), g(X)$ are 40 and 8. While, $f(X)$ and $g(X)$ are approached using 1403 and $556 \mathrm{RBF}$ neurons in [3] and [5] respectively. It is illustrated that the size of neural network is decreased greatly using the dynamic structure wavelet network.

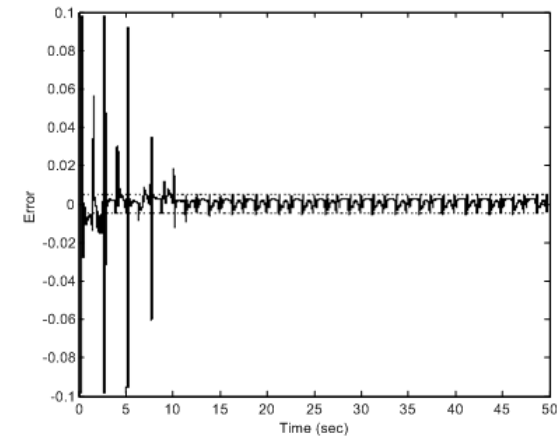

Fig. 1. Tracking Error; the dash line is the upper bound of tracking error.
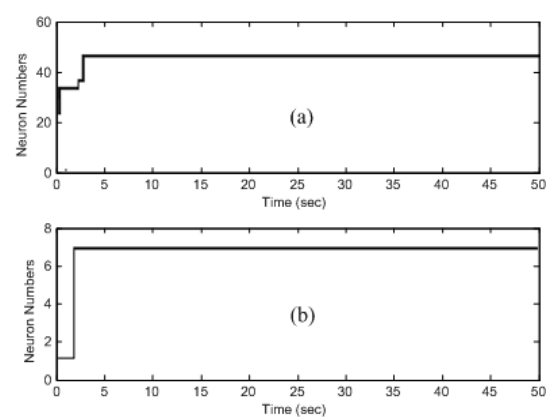

Fig. 2. The sizes of neural network for approximating $f(\mathrm{x}), g(x)$, (a) Neural Network for $f(x)$, (b) Neural Network for $g(x)$.

\section{References}

1. Yingwei $\mathrm{Lu}$ and P. Saratchandran, Identification of time-varying nonlinear systems using minimal radial basis function neural networks, IEE Proc.: Con. Theory and Appl. 144(2), pp. 202-208, 1997.

2. Cannon, M., Slotine, Jean-Jacques E., Space frequency Localized Basis Function Networks for Nonlinear System Estimation and Control, Neurocomputing, 9(3), pp.293342, 1995.

3. Fabri S., and Kadirkamanatham V., Dynamic Structure Neural Networks for Stable Adaptive Control of Nonlinear Systems. IEEE Transaction on Neural Networks, 7(5), pp.1151-1167, 1996.

4. Jeffrey T.S. and Kevin M. Passino, Stable Adaptive Control Using Fuzzy Systems and Neural Networks, IEEE Transactions on Fuzzy Systems 4(3), pp.339-359, 1996.

5. Sanner R.M. and Jean-Jacques E. Slotine, Gaussian Networks for Direct Adaptive Control, IEEE Transaction on Neural Networks, 3(6), pp.837-867, 1992.

6. Khalili ,H. Nonlinear systems, John Wiley \&Sons. NY, 1996.

7. Sastry S. and Alberto Isidori, Adaptive Control of Linearzable System, IEEE Transaction on Automatic Control, 34(11), pp.2247-2251, 1989.

8. Yesildirek A., Feedback Linearization Using Neural Networks, Automatica., 31(11), pp.1659-1664, 1995. 